\title{
Biomarkers of Adverse Response to Mercury: Histopathology versus Thioredoxin Reductase Activity
}

\author{
Vasco Branco, ${ }^{1,2}$ Paula Ramos, ${ }^{3}$ João Canário, ${ }^{2}$ Jun Lu, ${ }^{4}$ \\ Arne Holmgren, ${ }^{4}$ and Cristina Carvalho ${ }^{1}$ \\ ${ }^{1}$ Research Institute for Medicines and Pharmaceutical Sciences (iMed.UL), Faculty of Pharmacy, University of Lisbon, \\ Avenue Professor Gama Pinto, 1649-003 Lisbon, Portugal \\ ${ }^{2}$ Marine Environment and Biodiversity Unit, Institute for Sea and Atmospheric Research (IPIMAR/IPMA), \\ Avenue Brasília, 1440-006 Lisbon, Portugal \\ ${ }^{3}$ Aquaculture Unit, Institute for Sea and Atmospheric Research (IPIMAR/IPMA), Avenue Brasília, 1440-006 Lisbon, Portugal \\ ${ }^{4}$ Department of Medical Biochemistry and Biophysics, Karolinska Institutet, 17177 Stockholm, Sweden
}

Correspondence should be addressed to Cristina Carvalho, cristina.carvalho@ff.ul.pt

Received 20 April 2012; Accepted 25 May 2012

Academic Editor: João B.T. Rocha

Copyright ( 2012 Vasco Branco et al. This is an open access article distributed under the Creative Commons Attribution License, which permits unrestricted use, distribution, and reproduction in any medium, provided the original work is properly cited.

\begin{abstract}
Exposure to mercury is normally assessed by measuring its accumulation in hair, blood or urine. Currently, the biomarkers of effect that have been proposed for mercurials, such as coproporphyrines or oxidative stress markers, are not sensitive enough and lack specificity. Selenium and selenoproteins are important targets for mercury and thioredoxin reductase (TrxR) in particular was shown to be very sensitive to mercury compounds both in vitro and in vivo. In this study we looked into the relation between the inhibition of thioredoxin reductase (TrxR) activity and histopathological changes caused by exposure to mercurials. Juvenile zeabra-seabreams were exposed to $\mathrm{Hg}^{2+}$ or $\mathrm{MeHg}$ for 28 days and histopathological changes were analyzed in the liver and kidney as well as TrxR activity. Both mercurials caused histopathological changes in liver and kidney, albeit $\mathrm{Hg}^{2+}$ caused more extensive and severe lesions. Likewise, both mercurials decreased TrxR activity, being $\mathrm{Hg}^{2+}$ a stronger inhibitor. Co-exposure to $\mathrm{Hg}^{2+}$ and Se fully prevented TrxR inhibition in the liver and reduced the severity of lesions in the organ. These results show that upon exposure to mercurials, histopathological alterations correlate with the level of TrxR activity and point to the potential use of this enzyme as a biomarker of mercury toxicity.
\end{abstract}

\section{Introduction}

Adverse health effects of mercury include neurotoxicity, nephrotoxicity, cardiotoxicity, teratogenicity and immunotoxicity. However, the molecular mechanisms underlying mercury toxicity remain unclear with detrimental consequences on the development and validation of appropriate biomarkers of predictive toxic effects. Neurotoxic symptoms are the most visible aspect of mercury poisoning [1]. Nevertheless, the liver and kidney also accumulate high amounts of mercurials [2-4] that may impair their regular functioning. As happens with most xenobiotics, mercury compounds are mainly metabolized in the liver, where demethylathion $[5,6]$ and conjugation with Se $[7,8]$ or glutathione can occur [1]. In the liver of animals exposed to high levels of mercurials, hepatocytes are frequently hypertrophied with large-size vacuoles and widespread areas of necrosis can often be observed [3,9]. The proximal tubule is the kidney structure most affected by mercurials; the cellular changes include swelling of the mitochondrial matrix and endoplasmatic reticulum, loss of membrane integrity and eventual cellular necrosis [10]. Nephrotoxicity caused by to $\mathrm{Hg}^{2+}$ accumulation, is well recognized but may also arise from $\mathrm{MeHg}$ exposure [10, 11].

In risk assessment, biomarkers are important tools to assess the exposure, effect or susceptibility of individuals to a given xenobiotic. In the case of mercury compounds, only the use of biomarkers of exposure, such as the determination of mercury levels in hair $[12,13]$, blood $[14,15]$ and urine $[16,17]$ is generalized. However, the correlation between 
symptoms and $\mathrm{Hg}$ levels in hair, blood or urine is not always evident due to inter-individual variability in susceptibility to mercury [17] and to the delayed onset of effects that characterizes mercury poisoning [1]. Mercury toxic effects are frequently evaluated in humans and animals by conducting psychological and motor tests to assess the degree of neurological damage [17-19] although, changes in mental and motor skills might signify that mercury impairment of biological functions is already established. Therefore, the imposing challenge is still to identify a biomarker predictive of effect for mercury. Coproporphyrines levels and their excretion pattern in urine were proposed to evaluate early effects of mercurials $[20,21]$, but they have not proven to be a sensitive and useful indicator of mercury toxicity [19] and its use has been quite limited.

The affinity of mercury to thiol groups $(-\mathrm{SH})$ makes peptides and proteins vulnerable to its presence, especially when sulfhydryl groups are in the active site of enzymes. Changes in the activity of several enzymes involved in antioxidant action, such as glutathione reductase (GR) [22], superoxide dismutase (SOD) [23], and catalase (CAT) [24] are indicative of mercury induced oxidative stress. Glutathione depletion $[25,26]$, resulting from complexation with mercurials is also a good but unspecific indicator of mercury effects. Metallothionine induction [27] is a biochemical change that has been previously related to mercury exposure. Nevertheless, these changes are not specific and do not allow to distinguish mercury related effects in a multi-contaminant context.

Selenols $(-\mathrm{SeH})$ have a lower pKa than thiols (5.3 versus 8.5) and under physiological conditions are fully ionized to selenolates $\left(-\mathrm{Se}^{-}\right)$and thus are more reactive and can easily interact with mercury [28]. Selenoenzymes such as glutathione peroxidases (GPxs) are good targets for mercury [29-32] but, recently [28], the involvement of the thioredoxin system-comprising thioredoxin ( $\operatorname{Trx})$, the selenoenzyme thioredoxin reductase (TrxR) and NADPH-on the molecular mechanism of mercury toxicity was proven. The inhibitory effects of mercurials on the thioredoxin system have been shown both in vitro $[28,33]$ and in vivo $[4,34,35]$. Thioredoxin reductase is particularly sensitive to mercurials which results from its highly nucleophylic structure. Reduced TrxR has two active sites in each homodimer that include a dithiol in the FAD domain and a selenolthiol in the interface domain $[34,36]$. By contrast, the homologous enzyme GR, which differs from TrxR by lacking Se in the C-terminal active site, is not inhibited in the presence of mercury compounds [4, 28]. Given the importance of the $\operatorname{TrxR}$ and the thioredoxin system to several cellular functions such as protein repair and regulation of the cellular cycle [36], we hypothesize that TrxR inhibition might be a key mechanism by which mercury toxicity develops. Thus, this work investigates the incidence of histopathological changes in the liver and kidney of zeabra-seabreams and its correlation with the decrease in TrxR activity caused by $\mathrm{MeHg}$ or $\mathrm{Hg}^{2+}$ exposure. The influence of Se co-exposure and post-exposure treatment on enzyme activity and on the alterations observed is also discussed.

\section{Materials and Methods}

2.1. In Vivo Assays. Zeabra-seabreams (Diplodus cervinus), were used as a model. This species is easy to handle in captivity and, since the organs of fishes are similar to those of mammals, they constitute a good alternative to rodents as a model [37]. A total of 63 fishes were divided into 6 experimental groups: control $(\mathrm{C} ; n=12)$; selenium (Se, provided as sodium selenite; $n=9)$; exposure to $\mathrm{Hg}^{2+}$ ( $\mathrm{HgII} ; n=12$ ); exposure to $\mathrm{MeHg}(\mathrm{MeHg} ; n=12)$; coexposure to $\mathrm{Hg}^{2+}$ and Se (HgSe; $n=12$ ); co-exposure to $\mathrm{MeHg}$ and Se (MeHgSe; $n=12$ ). Juvenile zeabra-seabreams were kept in tanks at a density of $2.5 \mathrm{~g}$ of fish per liter. Oxygen saturation, in water was kept close to $100 \%$ and ammonia and $\mathrm{pH}$ were kept within normal limits. Exposure concentrations were set at $2 \mu \mathrm{g} \mathrm{L}^{-1}$ for both $\mathrm{Hg}^{2+}$ and $\mathrm{MeHg}$ and at $10 \mu \mathrm{g} \mathrm{L}^{-1}$ for Se. Exposure lasted 28 days and was followed by 14 days of depuration. During the depuration, fishes from $\mathrm{HgII}$ and $\mathrm{MeHg}$ groups were divided into two sub-groups, one kept in clean water and the other one kept in water supplemented with $\mathrm{Se}$ (HgRSe and MeHgRSe). The experiment was described in detail in Branco et al. [34].

2.2. Organ Collection. Sampling of fish took place at days 14, 28 and 42 (hereafter referred as d14, d28 and d42). Three fishes were taken from each group at each sampling day. The liver and kidney were collected and rinsed with $0.9 \%$ $\mathrm{NaCl}$. Sub-samples of these organs (were immediately fixed in $10 \%$ neutral buffered formalin (diluted in $10 \%$ salt water) for histopathological observation and the remaining organs frozen at $-80^{\circ} \mathrm{C}$ until analysis.

2.3. Histopathology Analysis. Following fixation in 10\% neutral buffered formalin, samples were washed with distilled water and dehydrated in a progressive series of ethanol, embedded in paraffin and then cut into $3 \mu \mathrm{m}$ thick sections further stained with Harris haematoxylin (Merck) and counterstained with Eosin (Merck) according to the standard method described by Lillie and Fullmer [39]. Histopathological observations were carried out by using a Olympus BX 51light microscope linked to a digital camera (Olympus DP-20).

The Organ Damage Index (ODI) was calculated for each exposure group at $\mathrm{d} 28$ and $\mathrm{d} 42$ according to the formula:

$$
\mathrm{ODI}=\sum_{n=1}^{4} \mathrm{Pi} \times N_{\mathrm{OL}}
$$

where $\mathrm{Pi}$ is the pathological importance factor following the proposal of Bernet et al. [38] for a given organ lesion (OL) observed in the number of fishes $(N)$ tested at each timepoint.

2.4. Total Protein Determination. Organ samples were homogenized with a glass mortar and Teflon pestle in TE buffer ( $\mathrm{pH} 7.5)$ containing a protease inhibitor cocktail (Roche), followed by centrifugation for $7 \mathrm{~min}$ at $12,000 \mathrm{rpm}$ and $4^{\circ} \mathrm{C}$. The pellet was discarded and supernatants used for enzyme activity assays. The total amount of protein in samples 


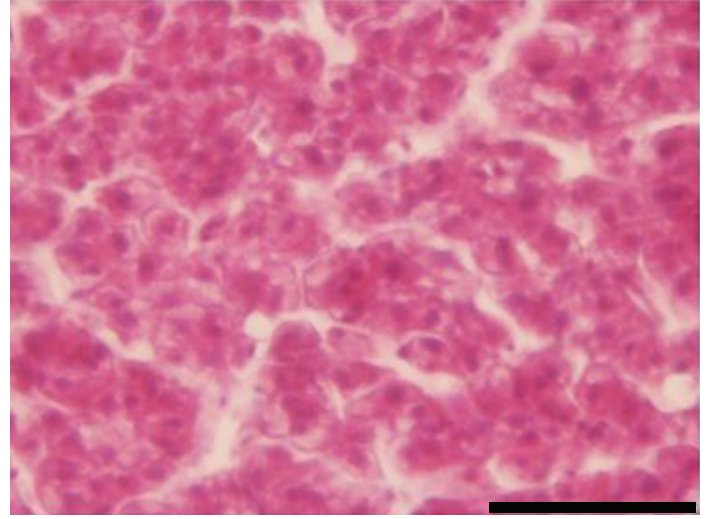

(a)

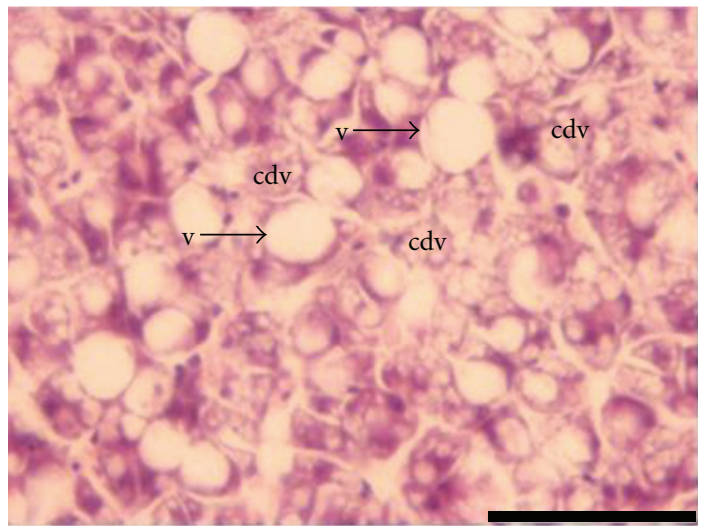

(c)

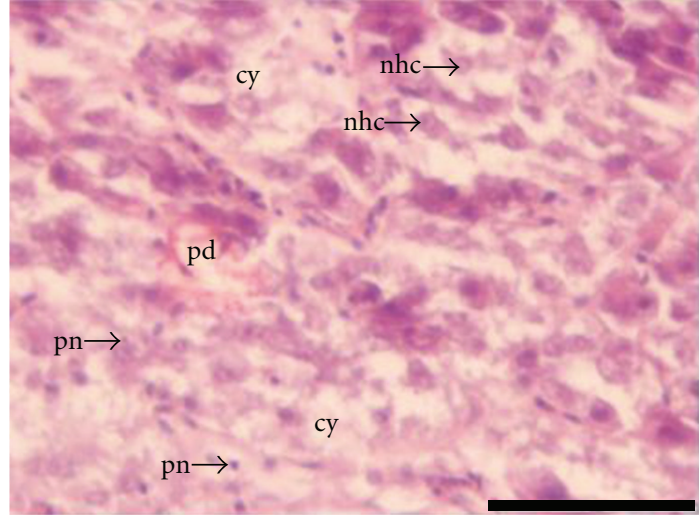

(b)

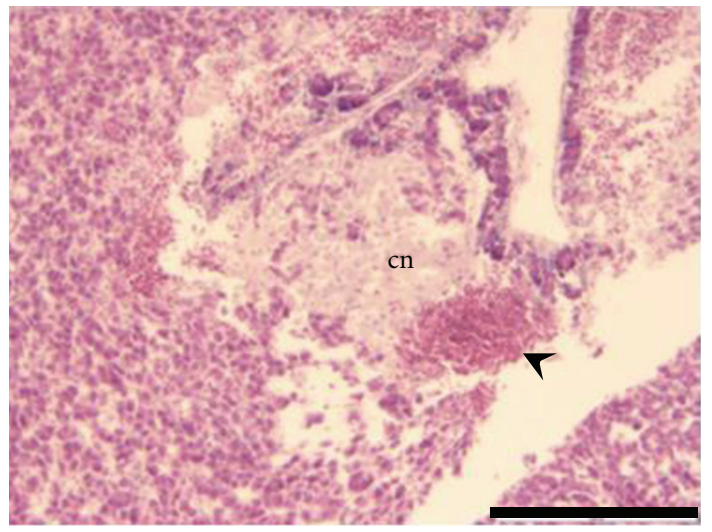

(d)

FIGURE 1: Histopathological observations in the liver of Diplodus cervinus after 28 days of exposure. (a) control group: section of polygonal hepatocytes cords (bar $=50 \mu \mathrm{m}$ ); (b) exposure to $\mathrm{Hg}^{2+}$ : extensive necrosis with congestion of sinusoids and pigment deposition (pd), nuclear hypercromatose (nhc), picnotic nucleus ( $\mathrm{pn}$ ) and cytolysis (cy) (bar $=50 \mu \mathrm{m})$; (c) exposure to MeHg: extensive cytoplasmic degenerative vacuolization (cdv) with large, smooth-edged vacuoles (v) (bar $=50 \mu \mathrm{m})$; (d) co-exposure to MeHg and Se: focal coagulative necrosis (cn) associated to blood congestion (arrowhead) (bar $=200 \mu \mathrm{m})$.

was determined in the supernatant fraction by measuring absorption at $595 \mathrm{~nm}$ in a microplate reader, according to Bradford [40], using Coomassie Brilliant Blue G-250 dye (Bio-Rad). Concentration of protein was quantified by using a calibration curve prepared by sequential dilution of a BSA standard solution.

2.5. Thioredoxin Reductase Activity. The activity of TrxR was determined using the insulin reduction endpoint assay proposed by Arnér and Holmgren [41]. Samples were incubated with TE buffer, fully reduced human $\operatorname{Trx}(3 \mu \mathrm{M}$; IMCO Corp. Sweden), insulin (0.3 mM), NADPH (2.5 mM), EDTA $(2.5 \mathrm{mM})$ and HEPES ( $85 \mathrm{mM}$; pH 7.6) for $20 \mathrm{~min}$ at room temperature. Control wells containing the same mixture but without added Trx were simultaneously prepared. After incubation, the reaction was stopped by adding $250 \mu \mathrm{L}$ of a $1 \mathrm{mM}$ DTNB solution in $6 \mathrm{M}$ guanidine $\cdot \mathrm{HCl}$ and absorbance at $412 \mathrm{~nm}$ was measured in a microplate reader.

2.6. Glutathione Reductase Activity. For GR activity, supernatants were incubated in 96-well plates with phosphate buffer (100 mM; pH 7.0), NADPH (1 mM), and GSSG $(200 \mu \mathrm{M})$. The reaction was monitored for $5 \mathrm{~min}$ at $30^{\circ} \mathrm{C}$ in a microplate reader and the decrease in absorption at $340 \mathrm{~nm}$, resulting from NADPH oxidation was registered [42].

2.7. Statistical Analysis. Differences between groups were assessed by computing the Mann-Whitney test for independent samples. Differences were considered significant at a $P$ value below 0.05 [43].

\section{Results and Discussion}

3.1. Liver Histopathology. Fish dissection showed that the liver of fishes from MeHg and HgII groups had softer consistency, when compared with the $\mathrm{C}$ group. No lesions or alterations were observed in the liver of controls (Figure 1(a)). The liver of fishes exposed for 28 days to $\mathrm{MeHg}$ and $\mathrm{Hg}^{2+}$ showed signs of hepatocyte alterations, namely, degenerative cytoplasm alterations, architectural pattern changes, loss of typical polygonal cell shape and undefined cell limits. Additionally, vacuolar degeneration with lateral migration of the 


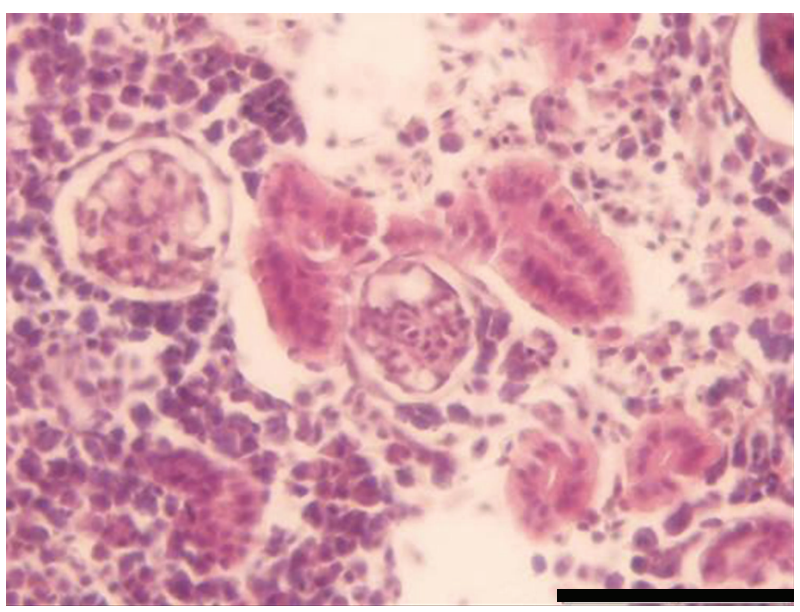

(a)

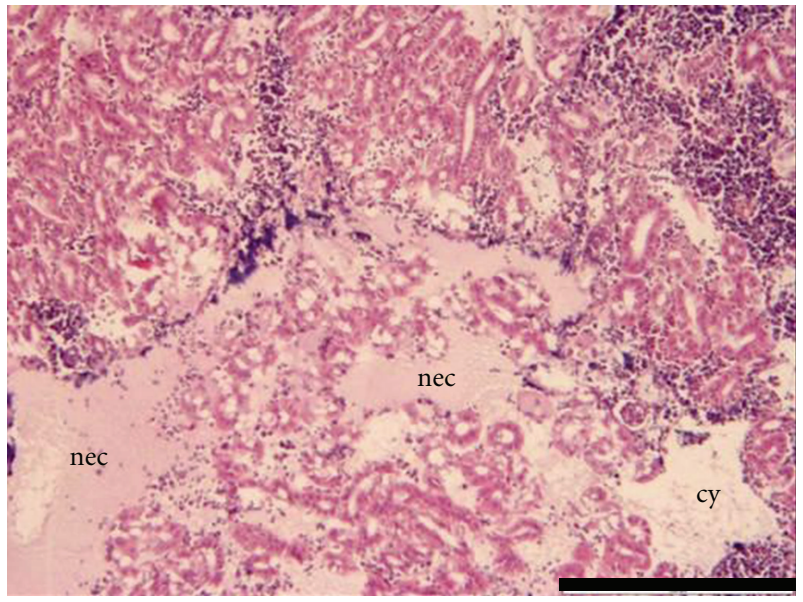

(b)

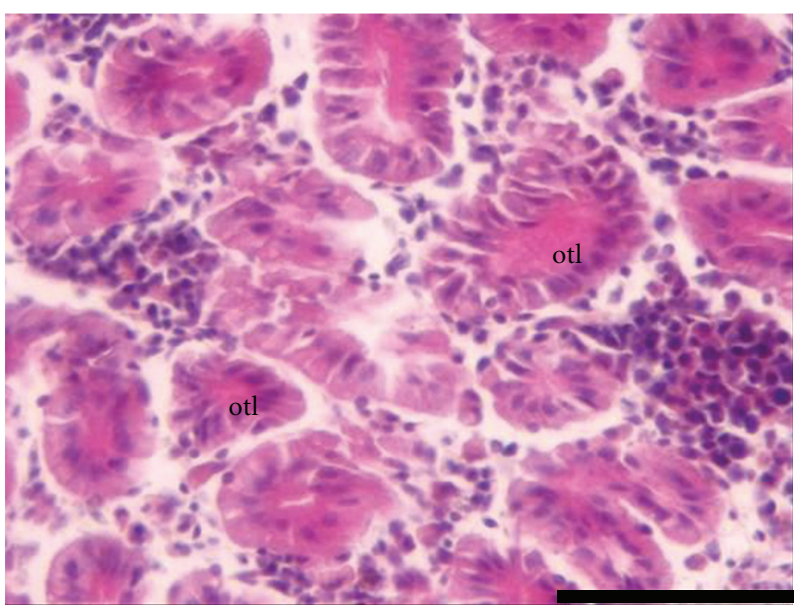

(c)

FIGURE 2: Histopathological observations in the kidney of Diplodus cervinus after 28 days of exposure to $\mathrm{Hg}^{2+}$. (a) control group: posterior kidney section $(\mathrm{bar}=50 \mu \mathrm{m})$; (b) exposure to $\mathrm{Hg}^{2+}$ : posterior kidney section showing a massive necrosis area $(\mathrm{nec})$ and cytolysis $(\mathrm{cy})(\mathrm{bar}=$ $200 \mu \mathrm{m})$. (c) exposure to $\mathrm{Hg}^{2+}$ : occlusion of the tubular lumen (otl) $($ bar $=50 \mu \mathrm{m})$.

nuclei, hydropic degeneration (Figure 1(b)), vacuolization within the hepatocytes with lipid-type vacuoles, which can be infiltrated fats, and appearance of some typical globular bodies may result from an increase in the lipid, water and/or glycogen content [3]. Hypertrophied hepatocytes were also disseminated at the parenchyma in fish from both HgII and $\mathrm{MeHg}$ groups, which is in good agreement with alterations described in the literature $[3,44,45]$. Liver extensive focal necrosis associated to congestion and pigments deposition was observed in two fishes belonging to the HgII group (Figure 1(c)). The MeHg group displayed three cases of little focal liver necrosis. During exposure to both mercurials the type of lesions was the same, but the necrotic lesions observed in the liver of fishes exposed to $\mathrm{Hg}^{2+}$ were more predominant and severe, leading to higher ODI values (Table 1). Despite the fact that, the accumulation of $\mathrm{Hg}^{2+}$ was lower than $\mathrm{MeHg}$ (Table 2), the ODI was higher. This result contrasts with the observations by Ribeiro et al. [45] where $\mathrm{Hg}^{2+}$ failed to cause any significant liver change in the artic charr, in comparison to MeHg. However, it should be stressed that Ribeiro et al. [45], used oral administration of mercurials with food and in those circumstances $\mathrm{Hg}^{2+}$, is much less absorbed in the GI tract than $\mathrm{MeHg}$ [1]. Although fishes from $\mathrm{HgSe}$ and $\mathrm{MeHgSe}$ groups (Figure 1(d)) showed at $\mathrm{d} 28$ the same kind of lesions observed in fish exposed only to mercurials (i.e, focal cellular vacuolization, megalocytic hepatocytes focal necrosis and congestion of the hepatic parenchyma), these lesions were observed side by side with normal cells.

After the depuration phase at $\mathrm{d} 42$, fish exposed to mercurials still exhibited extensive coagulative necrotic changes. Hypertrophy of the hepatocytes was clear in the parenchyma outside necrotic areas. Fishes depurating in water containing Se presented the same type of necrotic lesion although liver parenchyma beyond the necrotic zones appeared normal, resulting in a lower ODI than the index attained by fishes depurating in clean water (Table 1).

3.2. Kidney Histopathology. Kidney changes observed in fish exposed to both mercurials at days 28 and 42 were 


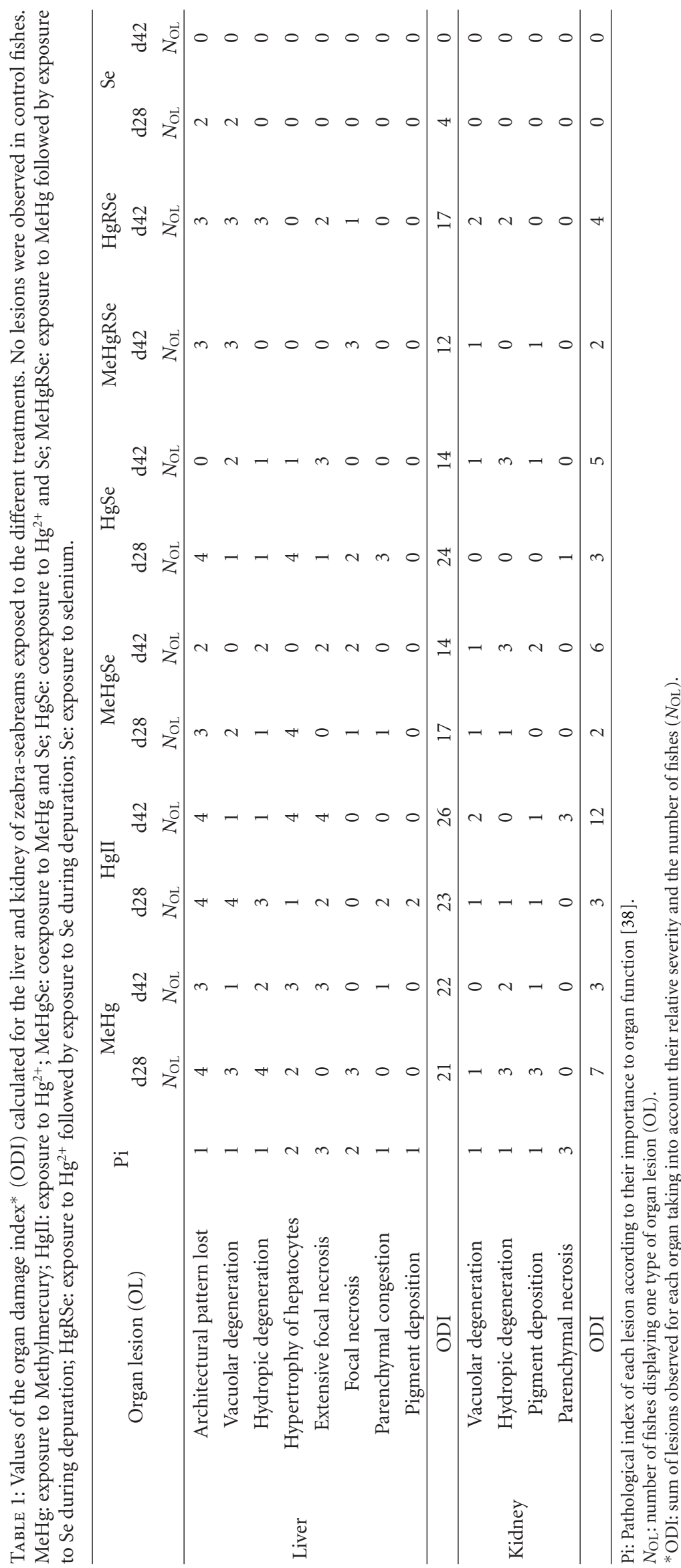




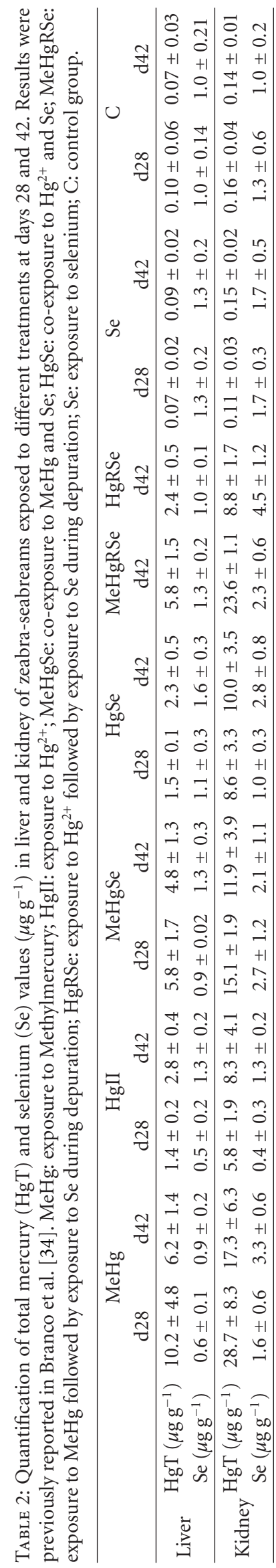




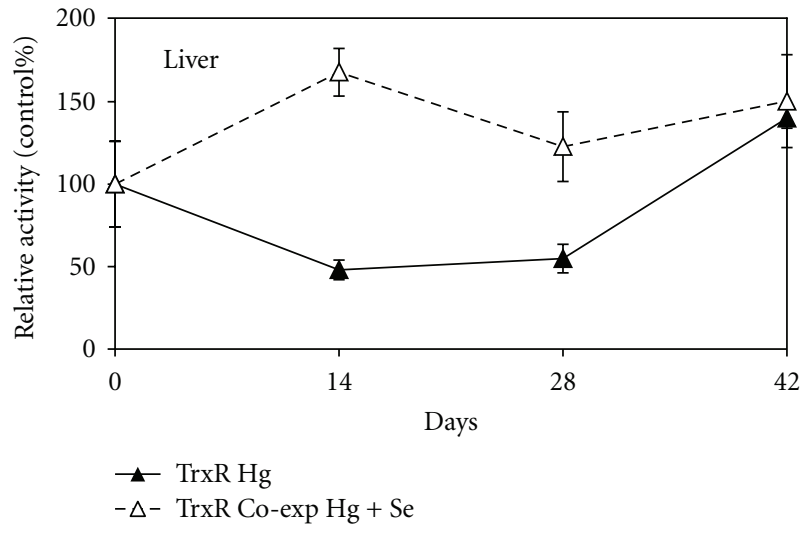

(a)

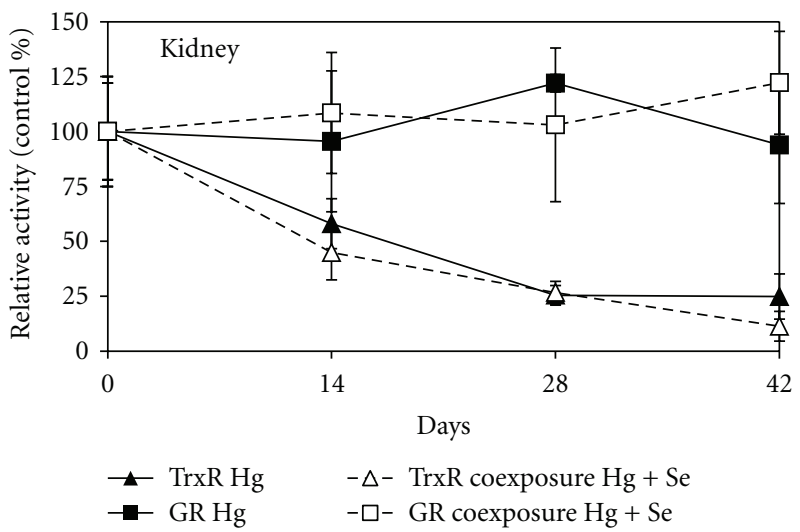

(c)

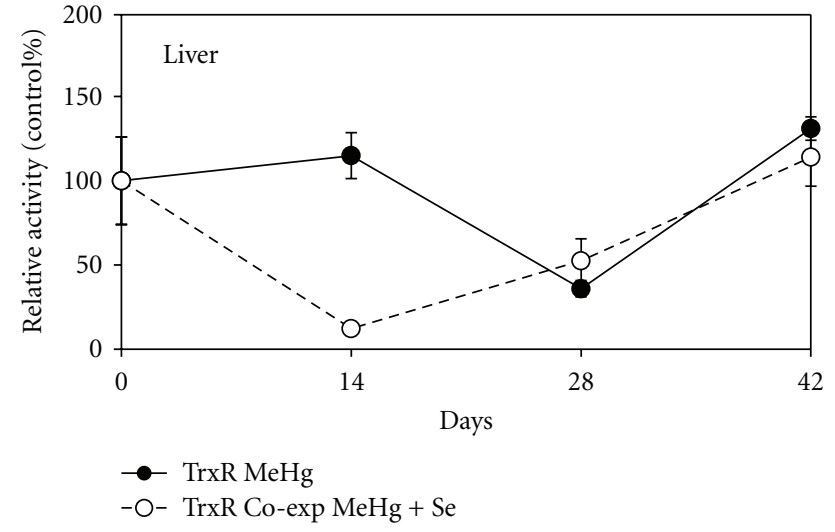

(b)

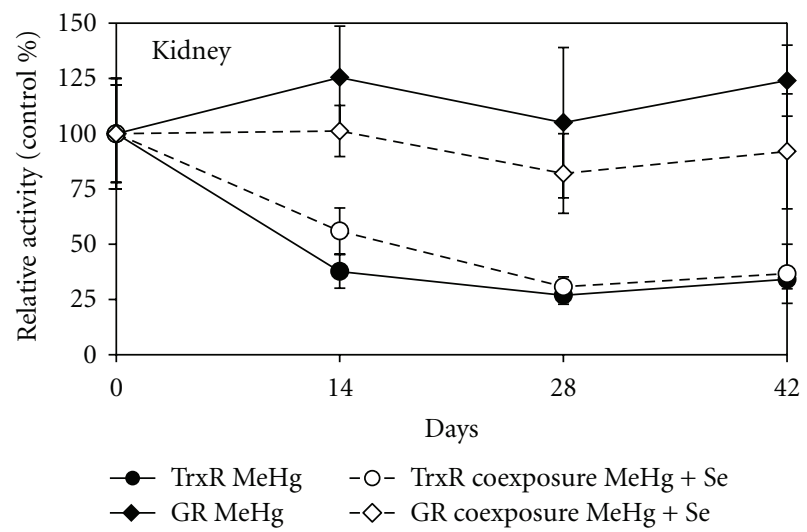

(d)

FIGURE 3: Enzymatic activities in liver and kidney of zeabra-seabreams exposed to mercurials and co-exposed to mercurials and Se (subset of results from a study previously reported in Branco et al. [34]). Exposure lasted 28 days and was followed by 14 days of depuration in clean or Se-supplemented water. (a) TrxR activity in the liver of seabreams exposed to $\mathrm{Hg}^{2+}$ and co-exposed to $\mathrm{Hg}^{2+}$ and Se; (b) TrxR activity in the liver of seabreams exposed to MeHg and co-exposed to MeHg and Se; (c) TrxR and GR activities in the kidney of seabreams exposed to $\mathrm{Hg}^{2+}$ and co-exposed to $\mathrm{Hg}^{2+}$ and Se; (d) TrxR and GR activities in the kidney of seabreams exposed to MeHg and co-exposed to MeHg and Se. GR activity in the liver did not show significant variation and was not represented to improve clarity.

vacuolar and hydropic degeneration of tubular epithelium and pigment deposits around the tubules. Comparatively, posterior kidney is quite more susceptible to $\mathrm{Hg}^{2+}$ which was evidenced by larger necrosis areas (Figure 2(b)) and occlusion of the tubular lumen with eosinophilic material (Figure 2(c)). Co-exposure to $\mathrm{MeHg}$ and Se seemed to delay the appearance of the more severe lesions in the kidney (Table 1). Co-exposure to $\mathrm{Hg}^{2+}$ and Se decreased the detrimental effects of mercury at the end of the depuration period (Table 1). This decrease in renal toxicity does not reflect a decrease in mercury concentration (Table 2) but instead it might be related to the formation of inorganic inert complexes.

3.3. Enzymatic Activities. The activity of TrxR in the liver and kidney of zeabra-seabreams is shown in Figure 3. In the liver, TrxR activity was decreased by $52 \%$ at $\mathrm{d} 14(P<0.05$; Figure 3(a)) during $\mathrm{Hg}^{2+}$ exposure, while in the case of $\mathrm{MeHg}$, the inhibition was only observed by $\mathrm{d} 28(P<0.05$; Figure 3(b)). As previously shown and discussed $[28,34,36]$ $\mathrm{Hg}^{2+}$ is a stronger inhibitor of TrxR than MeHg. Recovery of TrxR activity was complete at the end of depuration and was not influenced by Se supplementation during this period (data not shown). Co-exposure to Se besides reducing the ODI index, clearly prevented the inhibitory effect of $\mathrm{Hg}^{2+}$ over TrxR activity, that is, activity levels do not differ significantly from the control $(P>0.05$; Figure $3(\mathrm{a}))$, but was not effective over $\mathrm{MeHg}$ inhibition (Figure 3(b)) whose toxic effects seem to be increased by Se. In vitro experiments also confirmed that the co-exposure to $\mathrm{MeHg}$ and $\mathrm{Se}$ increased the detrimental effects over the TrxR activity [34]. At the same time it was shown that Se can remove $\mathrm{Hg}^{2+}$ bound to the selenolthiol in the active site of TrxR restoring activity while $\mathrm{MeHg}$ showed no displacement from the active site [34]. Further studies to fully elucidate the contradictory role of Se in the presence of different mercury species are being conducted.

In the kidney, TrxR activity was significantly affected $(P<0.05)$ both in $\mathrm{HgII}(42 \%$ inhibition) and $\mathrm{MeHg}(62 \%$ inhibition) groups at $\mathrm{d} 14$ and the inhibitory effect remained throughout the entire experiment (Figures 3(c) and 3(d)). Selenium showed no protective effect during co-exposure or 
on the recovery of TrxR activity during the depuration phase. As we have previously suggested [36], the protective Se over TrxR is organ specific and might be related to both the Se: Hg ratio and the capacity of selenoenzyme expression within the organ. In the liver, besides higher Se: Hg ratios [36] the level of selenoenzymes expression is assumed to be higher [44]. It should be stressed that, albeit TrxR activity did not recover, the citotoxic effects of $\mathrm{MeHg}$ decreased with $\mathrm{Se}$ coadministration in part due to the lower $\mathrm{MeHg}$ accumulation in both organs, liver and kidney, which reflected in the ODI values (Table 2). Since TrxR presents a high affinity for mercurials, any available mercury will primarily bound the selenolthiol of its active site and therefore it is normal that the inhibition of TrxR is observed in cases where cito-/organ toxicity is largely decreased (Table 1).

For the HgII group possible explanations for the decreased renal toxicity in the presence of Se include the formation of inorganic inert complexes and the participation of Se in antioxidant in cellular pathways. GR activity was not inhibited in the liver (results not shown) or in the kidney $(P>0.05$; Figures $3(\mathrm{c})$ and $3(\mathrm{~d}))$ in any group. On the contrary, the slight increase of GR activity seems to be a compensation mechanism for the loss of antioxidant protection provided by TrxR. Moreover, the fact that GR is homologous to TrxR, but lacks the Sec residue in the active site, reinforces the importance of TrxR active site as a main target for mercurials.

\section{Conclusions}

Mercury effects have been evaluated using different types of biomarkers. However, these are normally non-specific or are not predictive of toxicity but, instead, correspond to manifestations of toxicity itself. The thioredoxin system is responsible for several key cellular functions that range from anti-oxidant defense to regulation of the cellular cycle [33] and loss of activity will result in apoptosis [46]. Mercury (II) was shown to be a stronger inhibitor of TrxR than $\mathrm{MeHg}$ and also produced the most extensive array of organ lesions (Table 1). Also, when zeabra-seabreams were coexposed to $\mathrm{Se}$ and $\mathrm{Hg}^{2+}$ the severity of the lesions in the liver decreased while TrxR activity was kept at normal levels. In the cases of exposure to $\mathrm{Hg}^{2+}$ and $\mathrm{MeHg}$, although we observed full recovery of the activity of TrxR in the liver following depuration, the histopathological lesions reversion was not significant. Possibly, cellular mechanisms downstream of the thioredoxin system were affected to such a degree that recovery of TrxR activity does not reflect in an immediate organ recovery and time will be needed to replace damaged cellular structures. Overall, this work shows that the strong inhibition of TrxR activity is related with the histopathological alterations displayed in the liver and kidney of seabreams indicating the potential use of TrxR as a biomarker of effect of mercury toxicity.

\section{Acknowledgments}

The authors would like to thank Rui Silva and Pedro Pousão for assistance with the in vivo experiments. Vasco Branco was financed by a PhD fellowship (SFRH/BD/37388/2007) from the Fundação para a Ciência e Tecnologia (FCT) (http://www.fct.mctes.pt/). This study was financed by the MERTOX-TRX project (PTDC/QUI-BIQ/117281/2010) and by iMed.UL through FCT's strategic project: PEst-OE/SAU/ UI4013/2011.

\section{References}

[1] T. W. Clarkson and L. Magos, "The toxicology of mercury and its chemical compounds," Critical Reviews in Toxicology, vol. 36, no. 8, pp. 609-662, 2006.

[2] C. W. J. Chang, R. M. Nakamura, and C. C. Brooks, "Effect of varied dietary levels and forms of mercury on swine," Journal of Animal Science, vol. 45, no. 2, pp. 279-285, 1977.

[3] C. Liao, J. Fu, J. Shi, Q. Zhou, C. Yuan, and G. Jiang, "Methylmercury accumulation, histopathology effects, and cholinesterase activity alterations in medaka (Oryzias latipes) following sublethal exposure to methylmercury chloride," Environmental Toxicology and Pharmacology, vol. 22, no. 2, pp. 225-233, 2006.

[4] V. Branco, J. Canário, A. Holmgren, and C. Carvalho, "Inhibition of the thioredoxin system in the brain and liver of zebraseabreams exposed to waterborne methylmercury," Toxicology and Applied Pharmacology, vol. 251, no. 2, pp. 95-103, 2011.

[5] N. K. Mottet, M. E. Vahter, J. S. Charleston, and L. T. Friberg, Metabolism of Methylmercury in the Brain and Its Toxicological Significance, Marcel Dekker, New York, NY, USA, 1997.

[6] D. Yang, C. Yu-Wei, J. M. Gunn, and N. Belzile, "Selenium and mercury in organisms: interactions and mechanisms," Environmental Reviews, vol. 16, pp. 71-92, 2008.

[7] J. Parizek, I. Ostadalova, J. Kalouskva, A. Babichy, and J. Benes, The Detoxifying Effects of Selenium. Interrelation Between Compounds of Selenium and Certain Metals, Marcel Dekker, New York, NY, USA, 1971.

[8] M. A. K. Khan and F. Wang, "Mercury-selenium compounds and their toxicological significance: toward a molecular understanding of the mercury-selenium antagonism," Environmental Toxicology and Chemistry, vol. 28, no. 8, pp. 1567-1577, 2009.

[9] P. W. Wester and H. H. Canton, "Histopathological effects in Poecilia reticulata (guppy) exposed to methyl mercury chloride," Toxicologic Pathology, vol. 20, no. 1, pp. 81-92, 1992.

[10] R. K. Zalups, "Molecular interactions with mercury in the kidney," Pharmacological Reviews, vol. 52, no. 1, pp. 113-143, 2000.

[11] L. H. Lash, S. E. Hueni, D. A. Putt, and R. K. Zalups, "Role of organic anion and amino acid carriers in transport of inorganic mercury in rat renal basolateral membrane vesicles: influence of compensatory renal growth," Toxicological Sciences, vol. 88, no. 2, pp. 630-644, 2005.

[12] T. Giovanoli-Jakubczak, M. R. Greenwood, J. C. Smith, and T. W. Clarkson, "Determination of total and inorganic mercury in hair by flameless atomic absorption, and of methylmercury by gas chromatography," Clinical Chemistry, vol. 20, no. 2, pp. 222-229, 1974.

[13] H. Satoh, "Occupational and environmental toxicology of mercury and its compounds," Industrial Health, vol. 38, no. 2, pp. 153-164, 2000.

[14] J. C. Gage, "Distribution and excretion of methyl and phenyl mercury salts," British Journal of Industrial Medicine, vol. 21, no. 3, pp. 197-202, 1964.

[15] M. Berglund, B. Lind, K. A. Björnberg, B. Palm, Ö. Einarsson, and M. Vahter, "Inter-individual variations of human mercury 
exposure biomarkers: a cross-sectional assessment," Environmental Health: A Global Access Science Source, vol. 4, article 20, 2005.

[16] J. C. Gage, "The distribution and excretion of inhaled mercury vapour," British Journal of Industrial Medicine, vol. 18, no. 4, pp. 287-294, 1961.

[17] S. Bose-O'Reilly, K. M. McCarty, N. Steckling, and B. Lettmeier, "Mercury exposure and children's health," Current Problems in Pediatric and Adolescent Health Care, vol. 40, no. 8, pp. 186-215, 2010.

[18] T. Iwata, M. Sakamoto, X. Feng et al., "Effects of mercury vapor exposure on neuromotor function in Chinese miners and smelters," International Archives of Occupational and Environmental Health, vol. 80, no. 5, pp. 381-387, 2007.

[19] A. P. M. dos Santos, M. L. Mateus, C. M. L. Carvalho, and M. C. C. Batoréu, "Biomarkers of exposure and effect as indicators of the interference of selenomethionine on methylmercury toxicity," Toxicology Letters, vol. 169, no. 2, pp. 121-128, 2007.

[20] J. S. Woods, Porphyrin Metabolism as Indicator of Metal Exposure and Toxicity, Springer, Berlin, Germany, 1995.

[21] J. S. Woods, M. D. Martin, B. G. Leroux et al., "Urinary porphyrin excretion in children with mercury amalgam treatment: findings from the Casa Pia children's dental amalgam trial," Journal of Toxicology and Environmental Health A, vol. 72, no. 14, pp. 891-896, 2009.

[22] S. Oh and M. Lee, "Interaction between inorganic mercury and selenium on tissue sulfhydryl groups and glutathionelinked enzymes in rats," Yonsei Medical Journal, vol. 22, no. 2, pp. 122-126, 1981.

[23] F. Livardjani, M. Ledig, P. Kopp, M. Dahlet, M. Leroy, and A. Jaeger, "Lung and blood superoxide dismutase activity in mercury vapor exposed rats: effect of $\mathrm{N}$-acetylcysteine treatment," Toxicology, vol. 66, no. 3, pp. 289-295, 1991.

[24] R. Perrin-Nadif, M. Dusch, C. Koch, P. Schmitt, and J. M. Mur, "Catalase and superoxide dismutase activities as biomarkers of oxidative stress in workers exposed to mercury vapors," Journal of Toxicology and Environmental Health A, vol. 48, no. 2, pp. 107-119, 1996.

[25] T. Sarafian and M. A. Verity, "Oxidative mechanisms underlying methyl mercury neurotoxicity," International Journal of Developmental Neuroscience, vol. 9, no. 2, pp. 147-153, 1991.

[26] B. O. Lund, D. M. Miller, and J. S. Woods, "Studies on Hg(II)induced $\mathrm{H}_{2} \mathrm{O}_{2}$ formation and oxidative stress in vivo and in vitro in rat kidney mitochondria," Biochemical Pharmacology, vol. 45, no. 10, pp. 2017-2024, 1993.

[27] M. G. Cherian and T. W. Clarkson, "Biochemical changes in rat kidney on exposure to elemental mercury vapor: effect on biosynthesis of metallothionein," Chemico-Biological Interactions, vol. 12, no. 2, pp. 109-120, 1976.

[28] C. M. L. Carvalho, E. Chew, S. I. Hashemy, J. Lu, and A. Holmgren, "Inhibition of the human thioredoxin system: a molecular mechanism of mercury toxicity," The Journal of Biological Chemistry, vol. 283, no. 18, pp. 11913-11923, 2008.

[29] O. Wada, N. Yamaguchi, T. Ono, M. Nagahashi, and T. Morimura, "Inhibitory effect of mercury on kidney glutathione peroxidase and its prevention by selenium," Environmental Research, vol. 12, no. 1, pp. 75-80, 1976.

[30] C. Watanabe, K. Yoshida, Y. Kasanuma, Y. Kun, and H. Satoh, "In utero methylmercury exposure differentially affects the activities of selenoenzymes in the fetal mouse brain," Environmental Research, vol. 80, no. 3, pp. 208-214, 1999.

[31] C. Bulato, V. Bosello, F. Ursini, and M. Maiorino, "Effect of mercury on selenium utilization and selenoperoxidase activity in LNCaP cells," Free Radical Biology and Medicine, vol. 42, no. 1, pp. 118-123, 2007.

[32] J. L. Franco, T. Posser, P. R. Dunkley et al., "Methylmercury neurotoxicity is associated with inhibition of the antioxidant enzyme glutathione peroxidase," Free Radical Biology and Medicine, vol. 47, no. 4, pp. 449-457, 2009.

[33] C. M. L. Carvalho, J. Lu, X. Zhang, E. S. J. Arnér, and A. Holmgren, "Effects of selenite and chelating agents on mammalian thioredoxin reductase inhibited by mercury: implications for treatment of mercury poisoning," The FASEB Journal, vol. 25, no. 1, pp. 370-381, 2011.

[34] V. Branco, J. Canário, J. Lu, A. Holmgren, and C. Carvalho, "Mercury and selenium interaction in vivo: effects on thioredoxin reductase and glutathione peroxidase," Free Radical Biology and Medicine, vol. 52, no. 4, pp. 781-793, 2012.

[35] C. Wagner, J. H. Sudati, C. W. Nogueira, and J. B. Rocha, "In vivo and in vitro inhibition of mice thioredoxin reductase by methylmercury," BioMetals, vol. 23, no. 6, pp. 1171-1177, 2010.

[36] C. H. Lillig and A. Holmgren, "Thioredoxin and related molecules-from biology to health and disease," Antioxidants and Redox Signaling, vol. 9, no. 1, pp. 25-47, 2007.

[37] J. C. Wolf and M. J. Wolfe, "A brief overview of nonneoplastic hepatic toxicity in fish," Toxicologic Pathology, vol. 33, no. 1, pp. 75-85, 2005.

[38] D. Bernet, H. Schmidt, W. Meier, P. Burkhardt-Holm, and T. Wahli, "Histopathology in fish: proposal for a protocol to assess aquatic pollution," Journal of Fish Diseases, vol. 22, no. 1, pp. 25-34, 1999.

[39] R. D. Lillie and H. M. Fullmer, Histopathologic Technic and Practical Histochemistry, McGraw- Hill, New York, NY, USA, 1976.

[40] M. M. Bradford, "A rapid and sensitive method for the quantitation of microgram quantities of protein utilizing the principle of protein dye binding," Analytical Biochemistry, vol. 72, no. 1-2, pp. 248-254, 1976.

[41] E. S. J. Arnér and A. Holmgren, Measurement of Thioredoxin and Thioredoxin Reductase, John Wiley \& Sons, New York, NY, USA, 1999.

[42] B. Mannervik, Measurement of Glutathione Reductase Activity, John Wiley \& Sons, New York, NY, USA, 1999.

[43] J. H. Zar, Biostatistical Analysis, Prentice Hall, New Jersey, NJ, USA, 1999.

[44] M. Mela, M. A. F. Randi, D. F. Ventura, C. E. V. Carvalho, E. Pelletier, and C. A. O. Oliveira, "Effects of dietary methylmercury on liver and kidney histology in the neotropical fish Hoplias malabaricus," Ecotoxicology and Environmental Safety, vol. 68, no. 3, pp. 426-435, 2007.

[45] C. A. O. Ribeiro, L. Belger, E. Pelletier, and C. Rouleau, "Histopathological evidence of inorganic mercury and methyl mercury toxicity in the arctic charr (Salvelinus alpinus)," Environmental Research, vol. 90, no. 3, pp. 217-225, 2002.

[46] M. Conrad, "Transgenic mouse models for the vital selenoenzymes cytosolic thioredoxin reductase, mitochondrial thioredoxin reductase and glutathione peroxidase 4," Biochimica et Biophysica Acta, vol. 1790, no. 11, pp. 1575-1585, 2009. 

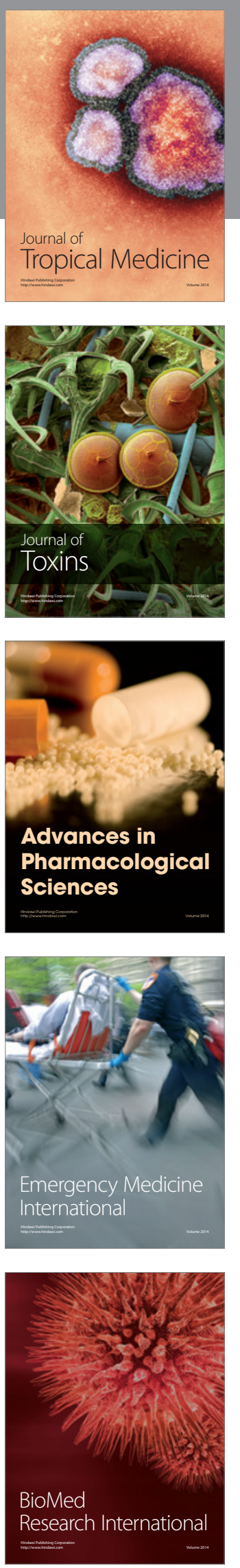
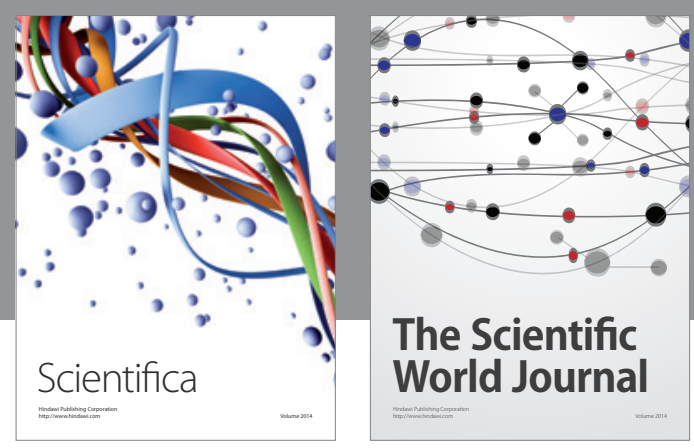

The Scientific World Journal
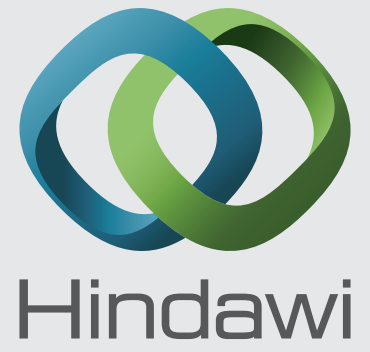

Submit your manuscripts at

http://www.hindawi.com
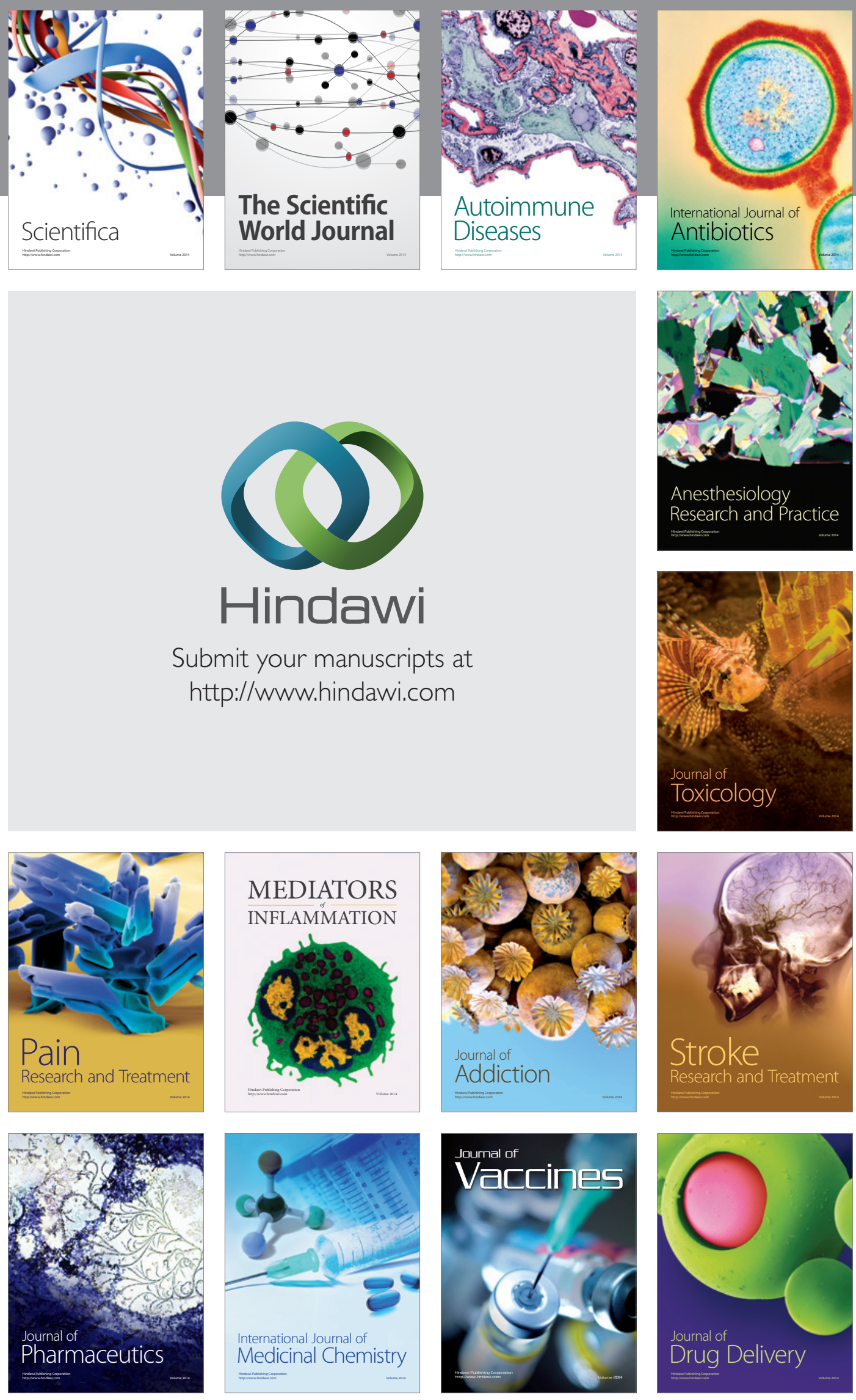